


\title{
EL TELETRABAJO, VENTAJAS E INCONVENIENTES
}

\author{
Autor: Jorge Navarrete Rojas ${ }^{1}$ \\ Gerente del departamento Finance \& Risk. Accenture
}

\section{Resumen}

Las transformaciones que están teniendo lugar en los últimos años de la mano de los crecientes avances tecnológicos plantean la necesidad de reflexionar acerca de los cambios en las formas de trabajo, es por ello que surgen a partir de finales del siglo XX una nueva forma de trabajo cuya característica principal es que lleva a cabo el trabajo desde el propio domicilio, fuera del ámbito de la oficina mediante la utilización de las tecnologías de la información y la comunicación (TIC), a esta nueva forma de trabajo se le conoce como teletrabajo.

Palabras clave: Teletrabajo; Acuerdo Marco Europeo sobre Teletrabajo; Tecnologías de la Información y el Conocimiento y del Comercio Electrónico (TIC); Teletrabajo en el domicilio, Telecentros u Oficinas satélite, Teletrabajo móvil; Oficina virtual.

${ }^{1}$ Doctorando del departamento de Gestión Empresarial Facultad de CC. EE. Y EE. Universidad Pontificia Comillas ICAI-ICADE. jnavarojas@yahoo.es 


\title{
Advantages and disadvantage of Telecommuting
}

\begin{abstract}
The transformations that are taking place in recent years in the hands of the growing technological advances suggest the need to reflect on the changing forms of work, which is why we emerge from the late twentieth century new ways of working which common link is carrying out the work from their own homes, outside the scope of the office through the use of information technology and communication (ICT), this new way of working is called telecommuting.
\end{abstract}

Key words: Telework; Telecommuting; European Framework Agreement on Telework, Information Technology and Knowledge and Electronic Commerce (ICT); telecommute at home, satellite offices or telecenters, Teleworking, Virtual Office.

\section{NUEVAS FORMAS DE TRABAJO}

El teletrabajo es un término tratado por diversas disciplinas científicas como la economía, la sociología o el derecho, que ha dado lugar a múltiples definiciones. Tal realidad y los riesgos de reduccionismo e imprecisión, han provocado que los autores tiendan a inclinarse por la descripción y análisis de los rasgos que lo identifican, en lugar de plantear una definición concreta del término. No obstante, existe una amplia variedad de definiciones entre las que se encuentran aquéllas que, describiendo al teletrabajo, nos ayudan a obtener esos rasgos que caracterizan al teletrabajo. A continuación, se recogen algunas de las principales definiciones de teletrabajo:

- Jack Nilles ya en 1976 lo definió como: "cualquier forma de sustitución de desplazamientos relacionados con la actividad laboral por tecnologías de la información" o de forma más gráfica "la posibilidad de enviar el trabajo al trabajador, en lugar de enviar el trabajador al trabajo".

- La Organización Internacional del Trabajo (1990), entiende por teletrabajo aquél que: "es efectuado en un lugar distante de la oficina central o del centro de producción e implica una nueva tecnología que permite la separación y facilita la información" 
- "Una forma flexible de organización del trabajo, que consiste en el desempeño de la actividad profesional sin la presencia física del trabajador en la empresa durante parte importante del horario laboral. Engloba una amplia gama de actividades y puede realizarse a tiempo completo o parcial. La actividad profesional en el teletrabajo implica el uso frecuente de métodos de procesamiento electrónico de información y el uso permanente de algún medio de telecomunicación para el contacto entre el trabajador y la empresa" (Gray, 1995).

A pesar de algunas de las definiciones que acabamos de ver, en este artículo nos quedaremos con la definición dada por la Oficina Europea de Estadística (EUROSTAT), que lo define como:

- Teletrabajo o trabajo desde casa como un concepto que se aplica a muchos trabajos autónomos, por ejemplo a profesiones artísticas o liberales cuyos trabajos se realizan total o parcialmente desde el hogar. Si el lugar de trabajo se encuentra separado del lugar donde se vive por una puerta de entrada (Ej: Sala de consulta de un doctor, Oficina de un contable) el trabajo realizado bajo estas premisas no se considerará teletrabajo.

De manera análoga un granjero no se considera teletrabajador cuando se ocupa de los campos o edificios adyacentes a su hogar.

En el caso de los empleados, el teletrabajo debe ser interpretado estrictamente en relación a un acuerdo formal con el empresario, en el cual ambas partes, están de acuerdo en que parte de la jornada laboral se realiza desde el hogar. Por tanto, el acuerdo debe estar explícitamente recogido en el contrato o reconocido de otra manera. Para ello es también necesario que el trabajador disponga de un ordenador para desarrollar su trabajo. Ejemplos típicos de teletrabajo incluye a los representantes de ventas quienes preparan en su domicilio para los mítines / discursos que tienen que llevar a cabo con los clientes en sus oficinas o en sus hogares, o las personas que mecanografían o puntúan trabajos.

El teletrabajo no cubre a los empleados que deciden trabajar en casa por motivos personales o razones de emergencia y cuyos contratos estipulan que tienen que realizar su trabajo desde su oficina.

Una persona es considerada como teletrabajador si en un periodo de cuatro semanas con anterioridad a la entrevista, él o ella ha trabajado desde casa bajo este tipo de acuerdo descrito anteriormente siendo el número de horas totales trabajadas de esta forma al menos de la mitad de las horas trabajadas durante este periodo.

Se la considera teletrabajador ocasional si en el periodo de referencia, cuatro semanas con anterioridad a la entrevista, él o ella han trabajado bajo el tipo de acuerdo comentado anteriormente, menos de la mitad de las horas totales. 


\section{Jorge Navarrete Rojas}

Una persona será no teletrabajador si en el periodo de referencia de cuatro semanas anteriores a la entrevista, él o ella nunca han trabajado desde casa bajo el tipo de acuerdo descrito anteriormente.

Una de las clasificaciones más comunes para definir el teletrabajo viene dada en función del lugar donde se desarrolla el trabajo, a continuación se presentan las diferentes tipologías de teletrabajo que existen en la actualidad:

- Teletrabajo en el domicilio. Esta modalidad representa la forma "clásica" de teletrabajo, donde las tareas se desarrollan en el seno del hogar y donde existe algún tipo de comunicación a distancia con el centro de trabajo tanto para recibir las órdenes como para enviar el resultado del trabajo. Algunos autores denominan a esta modalidad "teletrabajo a domicilio". En esta variante los teletrabajadores sustituyen la oficina o el centro de trabajo de la empresa para la que prestan sus servicios por su propio domicilio, como lugar donde llevar a cabo el trabajo habitual.

- Telecentros u oficinas satélites. En este supuesto el trabajador no se desplaza de su domicilio a la sede de la empresa, sino que lo hace a un centro específicamente diseñado para el desarrollo del teletrabajo. Se trata de lugares de trabajo con recursos compartidos y con instalaciones informáticas y de telecomunicaciones necesarias para llevar a cabo la prestación y en los cuales se suele enseñar a la población local a manejar esos recursos y a trabajar con ellos.

En los países en desarrollo, los telecentros brindan la oportunidad de utilizar las tecnologías de la información y de la comunicación con fines sociales, por ejemplo, en la enseñanza a distancia y la telemedicina.

- Teletrabajo móvil. Los teletrabajadores móviles son aquellos que pasan la mayor parte de su tiempo fuera de la oficina, ya sea en carretera o en las oficinas de los clientes. Normalmente se trata de agentes de ventas, técnicos o consultores. La oficina base de un teletrabajador móvil puede ser su casa, una oficina convencional, o incluso un vehículo. Este tipo de teletrabajo puede ser considerado también como trabajo independiente de una ubicación fija. Estos trabajadores constituyen uno de los grupos de teletrabajadores más numerosos y mejor asentados en todo el mundo, a pesar del hecho de que la mayor parte de ellos no se consideran a sí mismos como teletrabajadores.

- Oficina Virtual. Es un espacio virtual en el que pueden desarrollarse actividades similares a las de una oficina. Es decir, un lugar donde se desarrollan tareas profesionales con el fin de prestar un servicio a los clientes. Permite la expansión del lugar de trabajo en el tiempo y el espacio, dado que el trabajo está unido al trabajador, y no al flujo de papeles en un escritorio, de manera que el trabajo se puede realizar dondequiera que el trabajador se encuentre. 


\section{EL TELETRABAJO. VENTAJAS E INCONVENIENTES}

Son numerosas las ventajas, tanto para el trabajador como para la empresa y la sociedad a la hora de la adopción del teletrabajo. En primer lugar, nos centraremos en las ventajas para el trabajador:

1. Podrá decidir dónde y cuándo trabajar, siempre que cumpla con los objetivos marcados, es decir, llevará a cabo una gestión propia del tiempo (Brodt y Verburg, 2007).

2. Conciliación real de la vida personal y profesional (Harris, 2003).

3. Estilo de vida más saludable (Reduce el estrés de los trabajadores, Aumento del número de horas de sueño, Reducción de las bajas por enfermedad, etc).

4. Mayor predisposición a aumentar su rendimiento (Nicholas Bloom y otros, 2013).

5. Reducción de costes (Gastos de dietas, Gastos derivados del mantenimiento del vehículo, Ruedas, Aceite, etc.).

6. Reducción del tiempo de desplazamiento al trabajo, el tiempo destinado al transporte hasta los centros de trabajo es un tiempo perdido que se detrae del tiempo de ocio de los trabajadores.

Al igual que para el trabajador, el desarrollo de esta nueva forma de trabajar no sería posible si las empresas no se beneficiaran de la adopción de estas técnicas, ya que les permite:

1. Posicionamiento innovador en el mercado (Pyöria, 2011).

2. Retención del talento y menor rotación laboral, el teletrabajo permite atraer a nuevos empleados y retener a empleados valiosos, manteniendo y mejorando su estado (Bentley y Yoong, 2000).

3. Reducción de costes, asociado al espacio destinado a oficinas por parte de las empresas (Watad y DiSanzo, 2000).

4. Aumento de la productividad (Nicholas Bloom y otros, 2013).

5. Reducción del riesgo operacional ligado a fenómenos exógenos Ej.: La gripe A, erupciones volcánicas, etc.

6. Menor absentismo laboral (Collins, 2005).

Pero además hay que tener en cuenta las ventajas para la sociedad que son:

1. Reducción de la contaminación, hoy en día, el sector del transporte genera casi un 25\% de las emisiones globales de CO2, siendo las pertenecientes al transporte rodado de un $11 \%$. (RACC, 2007).

2. Reducción de la congestión del tráfico. 
3. Desarrollo rural de la población, con el nuevo modelo las personas podrían vivir en cualquier zona del territorio, lo que ayudaría a la dinamización de zonas deprimidas o rurales y evitaría la masificación de las grandes ciudades. Por tanto, favorecería la cohesión social de los países.

4. Mayor flexibilidad de la oferta de trabajo al permitir la integración en él de determinados colectivos: estudiantes, mujeres, discapacitados.

Pero no todo son ventajas a la hora de la implantación de las técnicas de teletrabajo, también existen una serie de desventajas, en el ámbito de la empresa son las siguientes:

1. Inversión en TICs. El coste de la instalación e infraestructura necesaria para realizar el teletrabajo, tanto en la empresa como para el teletrabajador.

2. Estructura más descentralizada. El teletrabajo supone un cambio en la estructura organizativa de las empresas y con frecuencia se produce una pérdida de jerarquías, debido a la dispersión de los teletrabajadores y a la no existencia de una supervisión continua. (Nilles, 1998).

En cuanto a los inconvenientes para el teletrabajador, se citan los siguientes:

1. Aislamiento de la empresa. Son numerosos los autores que afirman que el teletrabajo lleva al aislamiento social y profesional. Por esto, es importante que se adopten las medidas necesarias para organizar reuniones regulares y encuentros informales desde las primeras etapas de planificación del teletrabajo. Otros estudios sostienen que el teletrabajo a tiempo parcial no incrementa este aislamiento, al mantener un régimen de presencia en la empresa (Grenier, J. y otros 1997).

2. Dificulta el trabajo en equipo. En la sociedad actual existe un creciente interés por el trabajo en equipo (Nandhakumar y Baskerville 2006).

3. Pérdida del sentimiento de pertenencia a la empresa. Los avances tecnológicos están comenzando a mitigar los inconvenientes de esta naturaleza gracias a los programas como la mensajería instantánea, Skype o WebEx. Estas tecnologías permiten que los empleados interactúan con el trabajo de una manera más tradicional. Puede haber implicaciones culturales, ya que el teletrabajo es visto en organizaciones centralistas cómo un potencial que puede erosionar las relaciones entre sus empleados al interactuar menos con los otros y con la dirección.

No es posible implementar fórmulas de teletrabajo para aquellos empleados cuyo trabajo implique un servicio directo con la clientela o que requieren supervisión directa (Rosenlund, 2010). Se plantea que los empleados en estos puestos 
que, son típicamente los de menor retribución salarial, no se suelen beneficiar de los acuerdos de teletrabajo, mientras que los empleados que ocupan puestos de alto rango con relativamente menos preocupaciones económicas pueden disfrutar de los beneficios del teletrabajo.

En cuanto a los inconvenientes para el trabajador que continúa trabajando en la oficina, se citan los siguientes:

Un grupo especial que se ve afectado por el fenómeno del teletrabajo debido a la ausencia de los teletrabajadores es el de aquellos empleados que se encuentran en su oficina. Deben regularmente coordinar tareas, reuniones y sesiones de trabajo, tienen que programar o estructurar mejor su trabajo para compensar la ausencia de los teletrabajadores (Cooper \& Kurland, 2002).

1. La frustración también puede aparecer cuando se tiene un número de personas clave en la empresa teletrabajando y surge la necesidad de tener reuniones con diferentes unidades/áreas/ departamentos, estas reuniones tienen que ser estructuradas y planificadas para dar cabida al teletrabajador. El estudio de Golden (2007) sugiere que la prevalencia de teletrabajadores en una oficina se asocia negativamente con la satisfacción del compañero de trabajo y que esta relación está influenciada por la cantidad de compañeros que teletrabajan y el número de interacciones cara a cara que existe con ellos.

2. Si es necesario cubrir a los teletrabajadores y por lo tanto asumir responsabilidades adicionales que amplían el alcance y la cantidad de su trabajo, los no teletrabajadores serán más propensos a sentir la tensión y las actitudes de la desigualdad, por no hablar de los celos (Furnas, 2000). Estas imposiciones pueden conducir a la disminución de los niveles de satisfacción y generar sentimientos de irritación y hasta resentimiento hacia los teletrabajadores, sobre todo porque los teletrabajadores pueden obtener beneficios personales al conciliar trabajo y familia, mientras que ellos mismos no lo hacen (Hill y otros, 1998). Las entrevistas realizadas revelaron que "la actitud de los compañeros de trabajo varió desde su total escepticismo sobre el teletrabajo, a la aceptación y el respeto, aunque de vez en cuando se daban sentimientos de envidia, celos o resentimiento. Los autores señalan que la probabilidad de que se produzcan problemas de productividad para los no teletrabajadores puede surgir cuando uno adopte la fórmula del teletrabajo para evitar interrupciones", que afecta a sus compañeros que trabajan desde la oficina.

3. Algunos estudios anteriores, revelaron que, además de los beneficios de la flexibilidad, reducción de tiempo de viaje y la posibilidad de conciliar el trabajo y la vida personal, algunos empleados también optan por trabajar fuera de la oficina para evitar interrupciones y distracciones. Al considerar el grupo de trabajo que no realiza teletrabajo, (Bailey y Kurland, 2002) sugieren que algunas personas ya no tienen a nadie para interrumpir, mientras que otros son continuamente interrumpidos por la ausencia de los teletraba- 


\section{Jorge Navarrete Rojas}

jadores. En su estudio sobre el impacto del teletrabajo en la comunicación dentro de la organización varios teletrabajadores indicaron que percibían de sus compañeros que no teletrabajaban que estaban celosos porque el teletrabajado les permitía trabajar desde casa. Teletrabajadores que tenían esta percepción indicaron que sus colegas a menudo trataron de hacer que se sientan culpables por trabajar desde casa. "Mientras estabas en casa, tuve que lidiar con todos los problemas de los clientes ", " me ocupo de la "mierda "y usted tiene que sentarse en el sofá y leer los documentos ", " me gustaría hacer un trabajo de análisis, ya lo sabes, pero alguien tiene que quedarse en la oficina y apagar los incendios!” (Baruch, 2001). Las ideas de Baruch sobre algunas de las repercusiones organizativas del teletrabajo sugieren que los celos entre los no teletrabajadores y sus compañeros que teletrabajan pone de relieve la perspectiva de la justicia organizacional, es decir, su percepción de la equidad distributiva del teletrabajo. La percepción de la equidad de la distribución del trabajo en la práctica del teletrabajo es probable que surja, ya que afecta a los empleados que no teletrabajan. La percepción de la distribución del trabajo y de la selección de los empleados para teletrabajar es motivo de preocupación. Otros autores afirman que la importancia de ser tratado justamente por la organización es la base de una importante teoría de la motivación en el trabajo. De acuerdo con ellos la justicia en la toma de decisiones políticas y prácticas es una consideración importante para las personas. En la medida en que el teletrabajo implica la posibilidad de experimentar una sensación de inequidad o injusticia para los no teletrabajadores, es necesario que haya sensibilidad en su aplicación por parte de la organización (Alexander y Ruderman, 1987).

También se sugiere que si los empleados creen que los procedimientos de la organización son justos, son más propensos a estar satisfechos y apoyan los objetivos de la compañía, incluso si no están contentos con los resultados que reciben. Los problemas de celos entre pares típicamente surgen de ideas falsas acerca de quién se está beneficiando de teletrabajo y cómo fueron seleccionados los participantes (Fenson y Hill, 2003). Por tanto, la actividad del teletrabajo afecta a toda la organización, no sólo a los empleados que se les permite trabajar a distancia es importante que las organizaciones entiendan las variables que permiten o inhiben la satisfacción en el trabajo de los no teletrabajadores.

El teletrabajo se ha convertido en un tema importante en las políticas del mercado de trabajo, al considerar las amplias posibilidades que ofrecen las TICs. Con el continuo avance de la integración económica mundial, las decisiones sobre la ubicación de los centros de trabajo depende de las consideraciones sobre los costes laborales y las necesidades del cliente. La colaboración y el trabajo en red a través de las fronteras de los distintos países se ha convertido en una fuente importante de competitividad empresarial y de menores costes de producción (Andriessen y Vartiainen, 2006). 


\section{LA LEGISLACIÓN EUROPEA SOBRE EL TELETRABAJO}

En Europa no fue hasta la Cumbre de Lisboa del año 2000, donde se promovió un Grupo de Alto Nivel para que evaluara las oportunidades y los desafíos de las relaciones laborales en un entorno cambiante, con el objetivo de identificar los elementos o factores que podrán transformase para prevenir problemas y gestionar adecuadamente el cambio.

La legislación europea varía de un país a otro, no existía en el año 2000 un marco de común aplicación de principios rectores por el que debía regirse. A modo de ejemplo podemos citar algunos códigos, leyes y convenios que existían:

a) De naturaleza legislativa: Ley Bassanini sobre el teletrabajo en la función pública (Italia de 16 de junio de 1998).

b) Códigos de conducta: código de conducta redactado por el Comité Consultivo sobre teletrabajo creado en 1998 por los Ministerios de Ciencia, Tecnología y Comercio (Irlanda).

c) Convenios colectivos: a modo de ejemplo: convenio marco relativo al personal de las autoridades locales (Dinamarca); los convenios y modelos empresariales propuestos por los sindicatos: British Gas (Reino Unido), IBM y Deutsche Telecom (Alemania), TCO (Suecia), CONFAPI con CGIL-CISL y el convenio UIL sobre el teletrabajo (Italia).

Estas circunstancias justificaban la creación de un documento único fruto del consenso, que viniera a ofrecer los principios sobre los que debía asentarse la regulación del teletrabajo. Con fecha de 23 de mayo de 2002 se aprobaba de manera definitiva este texto, denominándose: Acuerdo Marco Europeo sobre Teletrabajo, que fue firmado con fecha de 16 de julio del mismo año en Bruselas por los interlocutores sociales (Confederación Europea de Sindicatos CES, la Unión de las Industrias de la Comunidad Europea UNICE/ UEAPME y el Centro Europeo de la Empresa Pública CEEP).

Esta norma vio comenzar sus negociaciones el 12 de octubre de 2001 teniendo su inmediata continuación en el Consejo Europeo de Laeken de diciembre de 2001 y finalizando el 23 de mayo de 2002 con su aprobación definitiva. Con anterioridad a estos contactos fue la Comisión Europea la que tuvo la decisión de emprender las consultas formales tanto a los representantes de las empresas como de los trabajadores, en virtud de lo dispuesto en el apartado 2 del artículo 138 del Tratado. El tema sobre el que giraba tal consulta era el relativo a la modernización y a la mejora de las relaciones laborales. Así pues la Comisión inició la primera fase de esta consulta el 27 de junio de 2000, y la segunda el 16 de marzo de 2001 (Gil y Gil, 2002).

En el caso de España no existe una normativa específica reguladora del Teletrabajo y nos regimos en la actualidad por: 
- Estatuto de los Trabajadores y Convenios Colectivos de aplicación.

- Regulación relativa al trabajo a domicilio.

- Recomendaciones de la OIT.

- Códigos de Buenas Prácticas del Teletrabajo.

- Acuerdo Marco Europeo sobre teletrabajo (2002).

Existen básicamente dos formas de implementación del teletrabajo entre los distintos países europeos "ley dura" y "ley suave".

Una diferenciación importante sobre la aplicación del Acuerdo Marco Europeo sobre teletrabajo es si crea una ley "suave" o "dura" en los respectivos Estados miembros.

"Ley suave" es el término que se aplica a las medidas de la UE como directrices, declaraciones y opiniones. En contraste a los instrumentos de "Ley dura" como son reglamentos, directivas y decisiones. Por tanto, las medidas de "Ley suave" no son vinculantes para aquellos a quienes se dirige. Sin embargo, puede producir algunos efectos jurídicos y a veces se presenta como un instrumento más flexible en la consecución de objetivos políticos. En la tipología de (Marginson y Sisson, 2006), "Ley dura" implica unos derechos estándar y obligaciones, mientras regula cuestiones básicas, como el salario y el tiempo de trabajo se basa en sanciones, es completa y obligatoria. La "ley suave", por el contrario, implica disposiciones mínimas sólo, regula "cuestiones suaves" como el estrés en el teletrabajo, es incompleta, abierta y permisiva.

Se dio a cada uno de los estados miembros la elección de cómo incorporar el Acuerdo Marco Europeo a su propia legislación. Los 21 países que informaron de algún tipo de aplicación del acuerdo se pueden agrupar en tres grupos. La forma más extendida de aplicación del Acuerdo Marco Europeo ha sido a través de convenios colectivos.

En un primer grupo se encuentran nueve países que lo aplican a través de los convenios principalmente a nivel nacional o intersectorial. En un segundo grupo de seis estados miembros, se encuentran los que lo aplican a través de diversas formas de medidas 'voluntarias', como directrices mixtas, códigos y recomendaciones sobre teletrabajo que se basan en las propuestas a nivel nacional o intersectorial. $\mathrm{Y}$ un tercer grupo donde se encuentran otros seis estados miembros, en ellos se ha promulgado una legislación nacional para aplicar el Acuerdo Marco Europeo.

Seis estados miembros han señalado que no habían aplicado el Acuerdo Marco Europeo sobre teletrabajo: Bulgaria, Chipre, Estonia, Lituania, Malta y Rumania.

Para evaluar las vías de implementación del teletrabajo se utilizan dos ejes, el de autonomía (X) y el de tipología de ley (Y). Los procesos de ejecución autónoma implican prácticas de autorregulación sin la interferencia de terceros, es decir, del Estado. Una implementación dependiente, por el contrario, se basa en un grado variable de intervención del Estado, dando así la posibilidad de regulación externa. 


\section{Figura 1. Formas de Implementación del Teletrabajo entre los diferentes países de la Unión Europea}

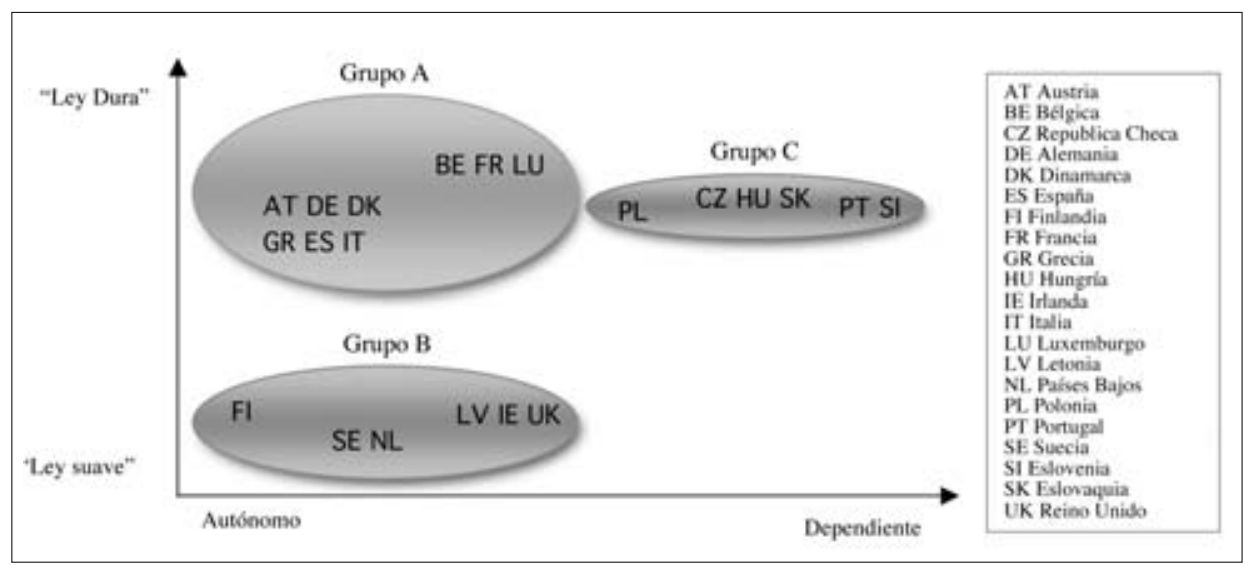

Fuente: Teletrabajo en la Unión Europea. European Foundation for the improvement of Living and Working condition, 2010.

Nota: Grupo C: Implementación a través de Legislación nacional. Grupo A: Implementación a través de convenios colectivos. Grupo B: Implementación a través de mecanismos de "Ley suave".

Dentro del grupo legislativo $(\mathrm{C})$, por ejemplo, pueden identificarse tres diferentes grados de autonomía versus dependencia. El impacto más fuerte de los interlocutores sociales en el proceso legislativo se llevó a cabo en Polonia, donde las regulaciones sobre el teletrabajo fueron introducidas en el código de trabajo como resultado de un acuerdo bilateral de los interlocutores sociales. En cierto modo, este modo de aplicación emula a nivel nacional la ampliación de la escala de la UE de un acuerdo social por decisión del Consejo. En la República Checa, Hungría y Eslovaquia, los interlocutores sociales nacionales fueron consultados por lo menos durante la aplicación legislativa. Consultas de la dirección y los trabajadores se llevaron a cabo en Portugal y Eslovenia. En Portugal, los interlocutores sociales al parecer no fueron muy favorables de la ruta de implementación estatutaria.

Dentro del grupo de convenio colectivo (A), también se observan diferentes grados de autonomía. Se han llevado a cabo convenios colectivos, evidentes en Austria, Dinamarca, Alemania, Grecia, Italia y España, pueden encontrarse en varios niveles de la estructura de las relaciones laborales. En el extremo más dependiente son los convenios colectivos nacionales, vistos en Bélgica, Francia y Luxemburgo, que se extienden por las autoridades públicas a todos los trabajadores. 


\section{Jorge Navarrete Rojas}

En el grupo de implementación a través de "Ley suave" (B), se encuentra el Reino Unido, el acuerdo de teletrabajo exigió negociaciones de nivel nacional entre el BIS (anteriormente el Departamento de comercio e industria) y el Congreso de Sindicatos (TUC). Los BIS no participaron en las negociaciones; sin embargo, según algunos comentaristas, facilitó las negociaciones y bajo la presión de los sindicatos británicos aceptó un código de prácticas como el instrumento adecuado (Deakin y Koukiadaki, 2007).

La mayoría de los estados miembros recurrió a los instrumentos de derecho duro, estatutos o convenios colectivos, en la aplicación del acuerdo de teletrabajo. El fundamento de este enfoque es evidente para que el Acuerdo sea aplicable a la mayoría de los trabajadores, los socios nacionales tuvieron que recurrir a un actor, el Estado, para ampliar el alcance del acuerdo a través de leyes o mecanismos de extensión.

Este enfoque implica una ventaja, pero también un inconveniente para los interlocutores sociales. Por un lado, se mejora la efectividad del Acuerdo autónomo aumentando el alcance. Por otra parte, se pierde parte de su autonomía, especialmente en el caso de la aplicación legislativa, ya que el gobierno es quién decide sobre el grado de incorporación de las disposiciones del Acuerdo. No es de extrañar que esta manera de implementación haya sido la principal vía de aplicación en los estados donde los actores y procesos de diálogo social siguen siendo bastante débiles a nivel nacional y, en particular, a nivel sectorial. En esos estados miembros, los interlocutores sociales nacionales han confiado en el estado para garantizar la plena vigencia del Acuerdo Europeo Autónomo (European Foundation for the Improvement of Living and Working Conditions, 2010).

En España, a día de hoy es importante mencionar que el teletrabajo se incluye en el texto de la Reforma Laboral 2012 (Real Decreto-ley 3/2012, de 10 de febrero, de medidas urgentes para la reforma del mercado laboral).

\section{LOS ACTUALES TELETRABAJADORES}

Para conocer el perfil tipo de los teletrabajadores, haremos referencia a diversos estudios, en el primero de ellos se nos dice que es un hombre de mediana edad con ingresos elevados, alto nivel educativo y profesionalmente independiente (Pérez y otros, 2001).

El perfil del teletrabajador a tiempo completo suele ser con una mayor probabilidad el de un hombre de mediana edad y con mayores ingresos, mientras que quienes aceptan teletrabajar a tiempo parcial son con mayor probabilidad las mujeres, algo más mayores y con menores ingresos (Cyber Dialogue, 2000).

Suelen ser candidatos idóneos los denominados "trabajadores del conocimiento", los trabajadores de la información, los vendedores y el personal de marketing. 
En relación con el status y el poder, el teletrabajo es más atractivo para los que ocupan puestos ejecutivos que para los simples empleados de oficina.

En relación con el perfil de las empresas que potencian las prácticas del teletrabajo no existe un consenso al respecto, mientras que existen, por ejemplo, compañías aéreas como JetBlue Airlines en dónde todos sus empleados de call center teletrabajan, en American Airlines por el contrario está prohibido.

Figura 2. Porcentaje de trabajadores que teletrabajan por tipo de trabajo

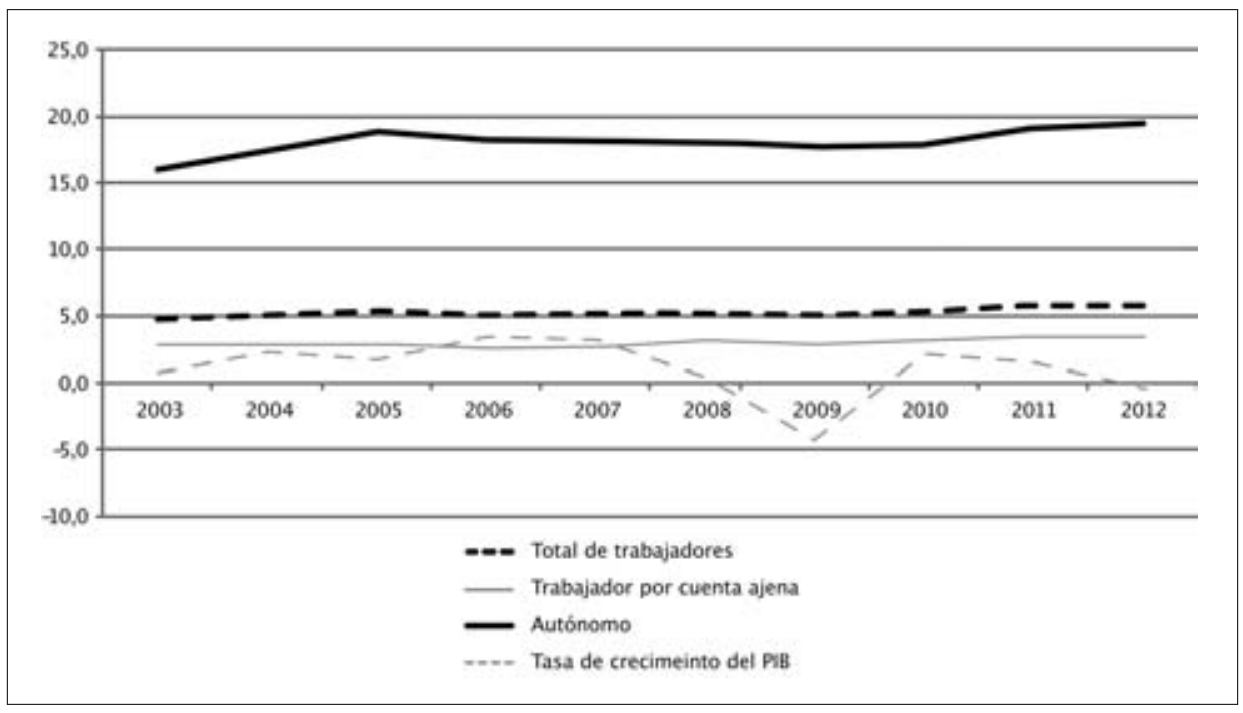

Fuente: EUROSTAT: Employed persons working from home as a percentage of the total employment, by sex, age and professional status (2003-2012).

A pesar, de la actual crisis económica el porcentaje de trabajadores que llevan a cabo fórmulas de teletrabajo de manera habitual ha seguido creciendo. Es de reseñar que en la actualidad cerca del 20\% de los trabajadores por cuenta propia teletrabajan, si nos centramos en los trabajadores por cuenta ajena esta cifra se reduce al 3,4\% en la UE-15.

Los adelantos en tecnología que nos permite trabajar de forma remota, se han desarrollado a un ritmo sin precedentes en las últimas dos décadas. Estos acontecimientos sólo no han reformado la estructura sectorial de la mayoría de las economías (el fenómeno denominado terciarización y el crecimiento de los sectores relacionados con el desarrollo y la producción de las TIC), sino también la manera en que se realizan innumerables tareas en muchas profesiones. 
Además, el número de tareas que no requieren de un lugar específico para su desempeño aumenta cada día debido a los avances tecnológicos. Esto significa que las formas de trabajo que se llevan a cabo están cambiando, así para algunas ocupaciones en una variedad de sectores el término 'teletrabajo' se ha utilizado durante muchos años para designar la manera de organizar o realizar el trabajo mediante el uso de las TIC.

En los Estados Unidos, aproximadamente el 10\% de los empleados trabajan desde casa al menos un día a la semana (Censo 2010), además la proporción que habitualmente teletrabaja se ha casi duplicado durante los últimos 30 años, del 2,3\% en 1980 al 4,3\% en 2010. Al mismo tiempo, el descuento en el salario por teletrabajar exclusivamente ha caído, del 30\% en 1980 a cero en el año 2000. Los teletrabajadores han pasado de estar centrados en empleos no cualificados a abarcar un amplio espectro de trabajos, que van desde gerentes hasta ingenieros de software (Oettinger, 2012) (ver figura 3).

El número de hogares en los Estados Unidos con ambos padres trabajando ha aumentado del 25\% en 1968 al 48\% en 2008 (Council of Economic Advisors, 2010), este hecho ha incrementado la presión y está llevando a los gobiernos de los Estados Unidos y Europa a investigar maneras de promover el equilibrio entre vida laboral y vida familiar.

\section{Figura 3. El teletrabajo es más común entre los grupos salariales más bajos y más altos}

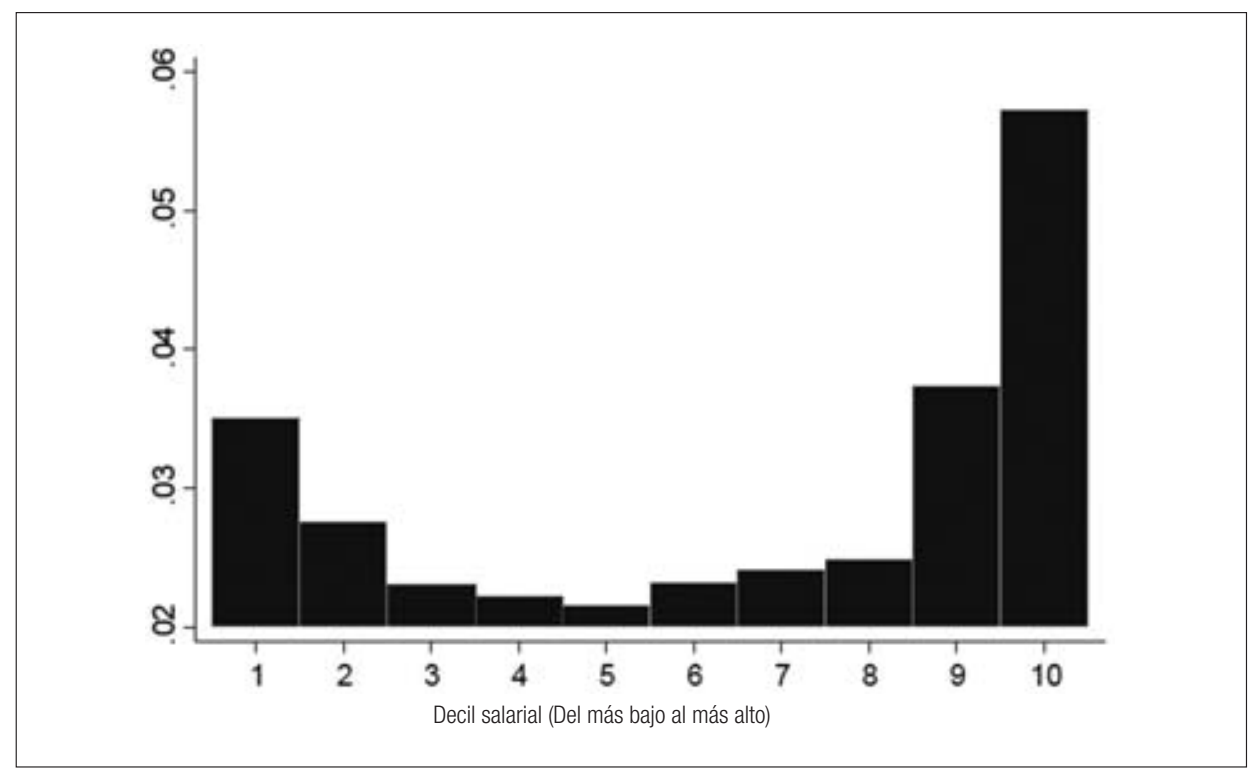

Fuente: Census Data, 2010. 
El principal problema que existe hoy día en España para el 75,3\% de la población es el paro, según los datos proporcionados por el CIS a septiembre de 2014.

El desempleo en España se sitúa en tasas cercanas al 24\% de la población activa según los datos proporcionados por Eurostat a agosto 2014. En datos absolutos supone que más de 5 millones de españoles quieren y no pueden trabajar.

Durante el año 2014, otros países de nuestro entorno han sido capaces de mantener tasas de desempleo inferiores al 10\%. El desempleo medio de la Unión Europea (UE-27) fue, según datos de Eurostat de Agosto 2014 es del 9,8\%.

Figura 4. Tasa de desempleo

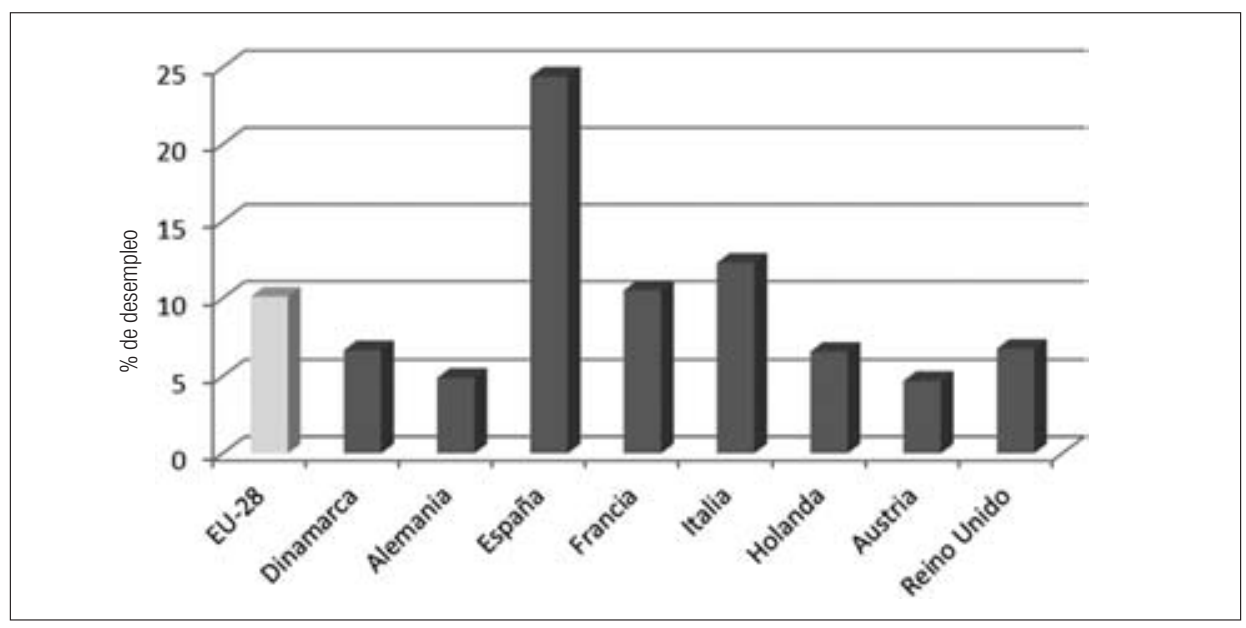

Fuente: Eurostat. Tasa de desempleo Agosto 2014.

Uno de los factores que afectan a esta elevada tasa de desempleo es sin duda la escasa implementación de técnicas de teletrabajo que ayudan a las empresas a reducir sus costes según (Dvorak y Anis, 1992). Para (Montiel, 2003) uno de los factores clave para la implementación del teletrabajo sobre todo en Italia es la reducción de costes que representa, para el caso español señala que la principal razón de su desarrollo es el aumento de la competitividad.

También (García Viñas, 2001), considera el teletrabajo como un instrumento de flexibilidad laboral que permite la externalización de las empresas y la reducción de sus costes fijos.

En España en los últimos 20 años hemos reducido en un 10\% el número medio de horas trabajadas (ver figura 5). Cada vez se valora más el ocio y por tanto existen claros incentivos a dedicar un menor número de horas al trabajo. El teletrabajo no reduce el número de horas de trabajo "per sé", lo que sí hace es reducir el tiempo que los trabajadores utilizan en desplazarse hasta su lugar de trabajo. 
Por tanto, la implementación del teletrabajo provoca que se aumente el tiempo de ocio de los trabajadores.

Además, cada trabajador podrá decidir cuándo trabajar, en función de las necesidades de la empresa y de su propia curva de producción (hay trabajadores que son más productivos a primera hora del día, otros sin embargo, prefieren las últimas horas de la tarde.

Figura 5. Jornada efectiva media por trabajador

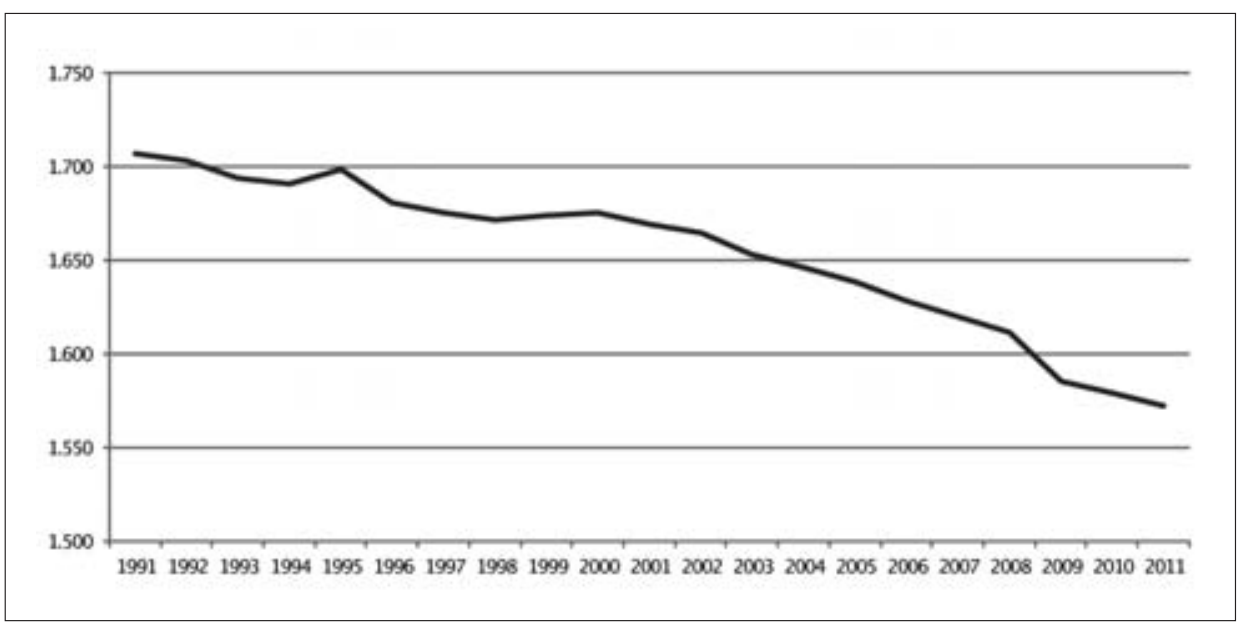

Fuente: INE. Condiciones de trabajo y relaciones laborales 1991-2011.

\section{PRINCIPALES VARIABLES A CONSIDERAR POR LAS EMPRESAS}

A la hora de implementar el teletrabajo es necesario que las empresas tengan en cuenta las siguientes características que han evolucionado en las últimas décadas:

- Precio de conexión.

- Calidad y velocidad de conexión.

Tabla 1. Evolución del coste relativo por mega en España

\begin{tabular}{|l|c|c|c|c|c|}
\hline & 2007 & 2009 & 2010 & 2011 & 2012 \\
\hline España & $13,30 \%$ & $6,82 \%$ & $6,82 \%$ & $4,09 \%$ & $4,09 \%$ \\
\hline
\end{tabular}

Fuente: Asociación de Internautas europeos. El ADSL en la UE: Una comparación (2012). 
Tabla 2. Infraestructuras TIC de las empresas

de menos de 10 empleados, en \%

\begin{tabular}{|c|c|c|}
\hline \multicolumn{3}{|c|}{$\begin{array}{l}\text { Infraestructuras TIC de las empresas de menos de } 10 \\
\text { empleados } \\
\text { Porcentajes }\end{array}$} \\
\hline & Enero de 2010 & Enero de 2011 \\
\hline Ordenadores & 66,2 & 69,7 \\
\hline Red de Área Local & 22,9 & 25,0 \\
\hline Red de Área Local sin hilos & 11,4 & 14,1 \\
\hline Conexión a Internet & 58,1 & 64,1 \\
\hline Conexión a Internet mediante banda ancha (fija o móvil)1 & 94,3 & 96,8 \\
\hline Telefonia móvil & 66,3 & 70,7 \\
\hline Otras tecnologias (p.e. GPS, TPV, etc.) & 15,5 & 17,9 \\
\hline$\%$ de empresas con conexión a Internet y sitio/página web. 1 & 25,0 & 25,9 \\
\hline
\end{tabular}

Fuente: INE. Encuesta sobre Equipamiento y Uso de Tecnologías de Información y Comunicación en los Hogares 2011.

- Cobertura para la conexión a Internet.

- Coste de los ordenadores.

El coste medio entre el año 2007 y el año 2012 ha bajado desde los algo más de 13 euros por mega hasta los $4 €$ por mega, esto supone un descenso considerable del precio por mega contratado del cerca del $70 \%$ en apenas 5 años.

El 69,7\% de las empresas españolas de menos de 10 empleados dispone de ordenadores y el 25,0\% tiene instalada una Red de Área Local (LAN). En cuanto al uso de Internet, el 64,1\% de las empresas pequeñas dispone de acceso a Internet, lo que supone un incremento del 10,4\% respecto a enero de 2010 . El 96,8\% de estas empresas con conexión a Internet acceden mediante alguna solución de banda ancha.

Según la última encuesta del INE sobre el uso de Tecnologías de la Información y el Conocimiento y del Comercio Electrónico en las empresas 2010/11 se desprende que:

- El 97,4\% de las empresas españolas de 10 o más empleados dispone de conexión a Internet.

- El 99,4\% de ellas se conecta por banda ancha (fija o móvil). 
Tabla 3. Índice de precios industriales. Fabricación de ordenadores y equipos periféricos

\begin{tabular}{|l|l|l|l|l|l|l|l|l|l|l|l|l|l|l|}
\hline & 2000 & 2001 & 2002 & 2003 & 2004 & 2005 & 2006 & 2007 & 2008 & 2009 & 2010 & 2011 & 2012 & 2013 \\
\hline $\begin{array}{l}\text { Fabrica- } \\
\text { ción de or- } \\
\text { denadores } \\
\text { y equipos } \\
\text { periféricos }\end{array}$ & 125,2 & 124,6 & 122,8 & 121,5 & 116,9 & 111,2 & 108,2 & 104,7 & 101,5 & 100,6 & 100 & 97,7 & 97,3 & 93,6 \\
\hline
\end{tabular}

Fuente: INE Índice de Precios Industriales 2013.

Las transformaciones que están teniendo lugar en los últimos años de la mano de los crecientes avances tecnológicos plantean la necesidad de reflexionar acerca de los cambios en las formas de trabajo.

Según el Índice de Precios Industriales, el precio de fabricación de ordenadores y equipos periféricos en España desde el año 2000 hasta finales del año 2013 ha sufrido un descenso de más de un 25\% (ver tabla 3 ).

\section{FACTORES QUE INFLUYEN EN LA IMPLEMENTACIÓN DEL TELETRABAJO EN EUROPA}

Como se verá más adelante existe una gran diferencia entre los porcentajes de implementación del teletrabajo entre los diferentes países europeos, para este trabajo se han seleccionado:

- Alemania

- Austria

- Dinamarca

- España

- Francia

- Holanda

- Italia

- Reino Unido

La suma de ellos supone casi un 70\% de la población europea (UE-27) a comienzos del 2011 según datos de EUROSTAT y es una muestra significativa del grado de implementación del teletrabajo en la Europa actual.

Entre estos ocho países existen dos grupos, en el primero de ellos se encuentran los países que no superan el 5\% de su población teletrabajando (Alemania, España, Italia y el Reino Unido), estos cuatro países suman casi el 50\% de la población total 
Figura 6. Porcentaje de implantación del teletrabajo en varios países europeos

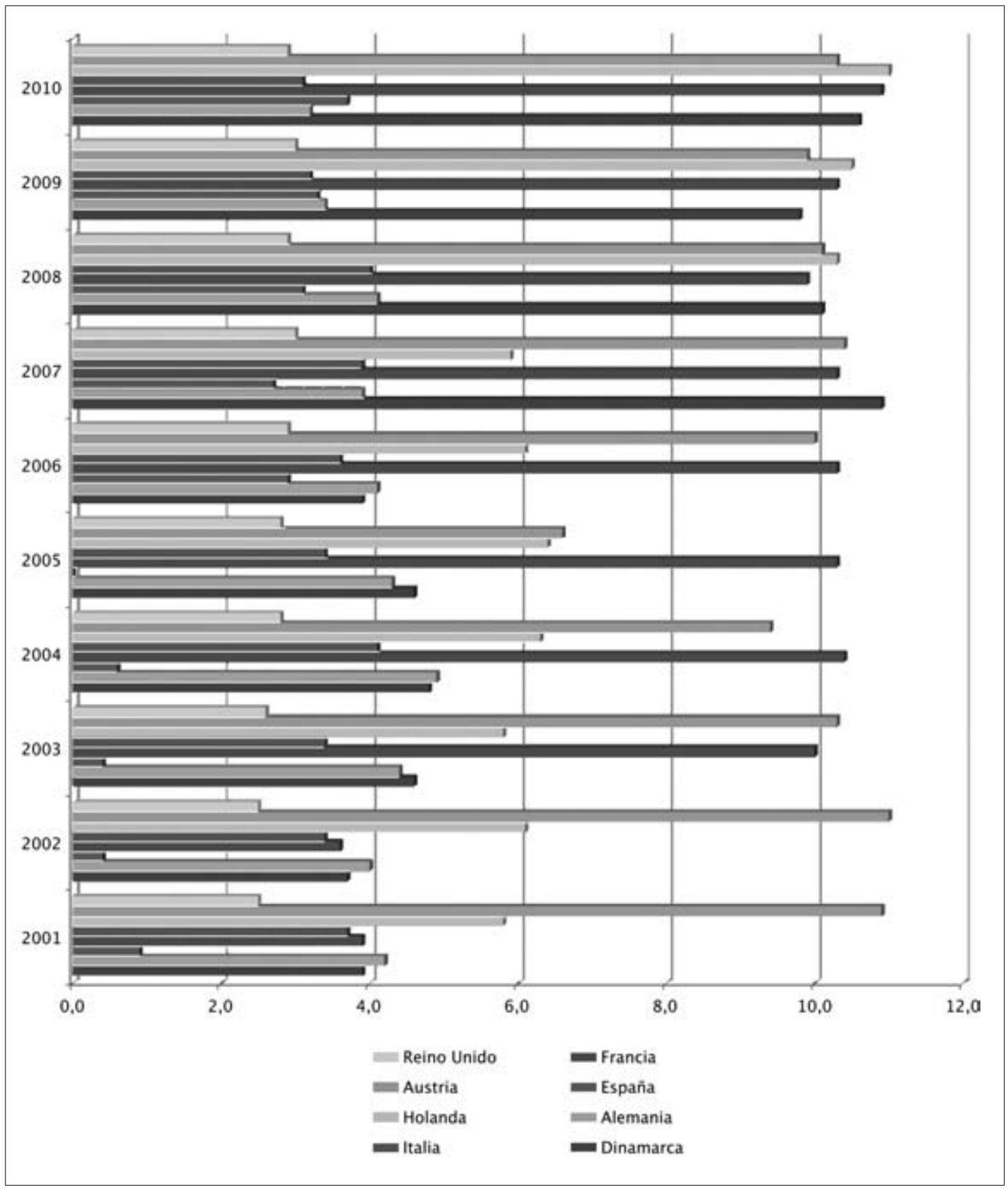

Fuente: EUROSTAT (2011).

icade. Revista cuatrimestral de las Facultades de Derecho y Ciencias Económicas y Empresariales, 
europea. En el segundo grupo, se encuentran aquellos países que superan el 10\% de población teletrabajadora, como son Francia, Dinamarca, Austria y Holanda, la suma de la población de estos países es de un 20\% del total europeo.

Es de destacar que la media de teletrabajadores en la UE a 25 se sitúa en el 5\% de la población ocupada. En el siguiente gráfico se muestra cuál ha sido la evolución del teletrabajo entre el año 2000 y 2010 en los países objeto de estudio.

\section{Tabla 4. Variables exógenas (explicativas)}

\begin{tabular}{|c|l|l|}
\hline Código & Variables explicativas (exógenas) & Fuente \\
\hline 1 & Porcentaje total de la población que ha acabado al menos los estudios secundarios & Eurostat \\
\hline 2 & Porcentaje total de la población que ha acabado al menos los estudios universitarios & Eurostat \\
\hline 3 & Gasto en educación & Eurostat \\
\hline 4 & Productividad del trabajo & Eurostat \\
\hline 5 & PIB per cápita & Eurostat \\
\hline 6 & Porcentaje de Trabajadores por cuenta ajena sindicalizados & OCDE \\
\hline 7 & Tasa de desempleo hombres & Eurostat \\
\hline 8 & Tasa de desempleo mujeres & Eurostat \\
\hline 9 & Coste por mega & Asociación de internautas \\
\hline 10 & Rendimiento de los alumnos en lectura & Informe PISA \\
\hline 11 & Rendimiento de los alumnos en matemáticas & Informe PISA \\
\hline 12 & Rendimiento de los alumnos en ciencias & Informe PISA \\
\hline
\end{tabular}

Fuente: elaboración propia.

A la hora de analizar qué variables son las que influyen en el porcentaje de personas que teletrabajan en cada uno de los países objeto de estudio se ha utilizado un modelo de regresión:

$$
\mathrm{Y}_{\mathrm{ct}}=\alpha+\beta \mathrm{X}_{\mathrm{ct}}+\lambda \mathrm{W}_{\mathrm{c}}+\xi_{\mathrm{ct}}
$$

Donde, Yct: es la variable dependiente (endógena), Xct: son las variables explicativas del modelo, $\beta$ es el parámetro a estimar y Wc.: Es la variable ficticia (dummy) y el término $\xi_{c t}$ indica el error cometido en la estimación de la variable dependiente, siendo $\alpha$ la constante.

Vamos a realizar una asunción fuerte que es que esta variable ficticia (dummy) no se encuentra correlada con Yct y Xct. Además, estas variables que se encuentran recogidas en la variable ficticia van a ser constantes en el tiempo (Por ejemplo: la fiscalidad de los distintos países).

Seis de las doce variables explicativas de este artículo se centran en variables educativas (nivel de estudios, gasto en educación, rendimiento de los alumnos), una en el coste de conexión a Internet y el resto se centran en 
variables de tipo macroeconómico, además de las incluidas podrían añadirse como variables exógenas:

- Estructura productiva de la economía.

- Situación del mercado laboral (flexibilidad de la oferta, flexibilidad de la demanda, situación de la tasa de actividad y de la tasa de desempleo por edades, nacionalidad o nivel de formación, etc.).

- Factores geográficos y climatológicos.

El modelo de regresión lineal es estadísticamente significativo para tres de las variables objeto de estudio (ver tabla 5) al 5\%. En ellos se establece una relación positiva entre el aumento del gasto en educación y el aumento del número de teletrabajadores, lo cual nos indica que un aumento en el gasto educativo mejoraría el porcentaje de teletrabajadores en un país, pero aun siendo esto cierto, el efecto de dicho aumento en el porcentaje de teletrabajadores tiene un efecto es muy reducido de solo un 0.01 .

Se establece una relación negativa entre el aumento en el número de trabajadores por cuenta ajena sindicalizados y el porcentaje de teletrabajo. Esto nos sugiere que una de las trabas que se tiene en las empresas para adoptar técnicas de teletrabajo viene de los propios representantes de los trabajadores que complican la implantación de esta nueva forma de trabajo.

Se establece también una relación positiva entre el rendimiento de los alumnos en matemáticas y la implantación del teletrabajo, este resultado nos indica que cuanto mejores son los resultados de los alumnos en matemáticas mayor predisposición se tiene por parte de estos a teletrabajar.

Uno de los inputs que las empresas no pueden controlar, ya que les viene dado, es el precio de conexión a Internet. Se establece una relación positiva entre el coste de conexión y el porcentaje de teletrabajo a un 10\%, lo que nos hace pensar que este no es un factor tan determinante para las empresas a la hora de implementar el teletrabajo.

El modelo de regresión utilizado presenta un R-cuadrado de 0.7815 , es decir, que el $78,15 \%$ de la variabilidad en el teletrabajo es explicada por las variables independientes analizadas.

A continuación, se muestran los resultados obtenidos en un modelo de efectos fijos (ver tabla 5), es un método lineal de estimación de datos de panel estático que considera $\alpha$ como un término constante específico del grupo, por tanto, en este segundo modelo los resultados muestran sólo el efecto de las variaciones que ha habido en cada país (Ej: España) en el periodo seleccionado 2000-2010, este método permite un mejor control de la heterogeneidad individual y permiten el uso de una mayor cantidad de información. Pero posee el inconveniente de no calcular el efecto de variables que no varían en el tiempo. 
Tabla 5. Resultado de la Regresión Lineal

\begin{tabular}{|c|c|c|c|c|c|c|}
\hline Teletrabajo & Coef. & Std. & Err. & $\mathbf{t}$ & $\mathbf{P}>|\mathbf{t}|$ & [95\% Conf. Interval] \\
\hline Estudio Secundaria & -.0116928 & .0700818 & -0.17 & 0.872 & -.1774099 & .1540243 \\
\hline Estudio Universitario & -.3156911 & .1426974 & -2.21 & $0.063 *$ & -.6531168 & .0217347 \\
\hline Gasto Educación & .0100757 & .0033978 & 2.97 & $0.021^{* *}$ & .0020413 & .0181101 \\
\hline Productividad & 7.277 .793 & 17.746 & 0.41 & 0.694 & -3.468 .527 & 49.240 \\
\hline PIB per capita & -1.438 .624 & 7.975 & -1.80 & 0.114 & -3.324 .547 & 4.472 \\
\hline Trabajadores Sindicalizados & -.1221796 & .0459768 & -2.66 & $0.033 * *$ & -.2308975 & -.0134616 \\
\hline Desempleo Hombres & .3209123 & .4668699 & 0.69 & 0.514 & -.7830596 & 1.424 \\
\hline Desempleo Mujeres & -.425021 & .3261198 & -1.30 & 0.234 & -1.196 .172 & .3461298 \\
\hline Coste por mega & .3674228 & .1842026 & 1.99 & $0.086 *$ & -.0681471 & .8029928 \\
\hline Resultado Lectura informe Pisa & -.1040046 & .0615275 & -1.69 & 0.135 & -.249494 & .0414849 \\
\hline Resultado Matemáticas informe Pisa & .088445 & .0345459 & 2.56 & $0.038 * *$ & .0067569 & .1701332 \\
\hline Resultado Ciencias informe Pisa & .0116953 & .0305736 & 0.38 & 0.713 & -.0605997 & .0839903 \\
\hline Constante & 38.413 & 72.308 & 0.53 & 0.612 & -132.569 & 209.397 \\
\hline
\end{tabular}

Significativo al: $* 10 \%$, Significativo al $* * 5 \%$, Significativo al $* * * 1 \%$.

Las variables (Productividad, PIB per capita y Coste por mega) se encuentran definidas en logaritmo.

Las diferencias entre los distintos países pueden ser captadas a través de las diferencias en dicho término constante, de modo que cada $\alpha$ es un parámetro desconocido a estimar.

En nuestro estudio hemos trabajado con un panel equilibrado. En este modelo se establece una relación negativa entre el número de personas con educación secundaria y el número de teletrabajadores a un nivel de significación del 1\%. El efecto de esta variable es fuerte al tener un coeficiente de -0.58.

Otra de las variables significativas al $1 \%$ es la tasa de desempleo femenina. A mayor tasa de paro de un país menor es el porcentaje de teletrabajadores". Si bien es cierto que solo se cumple para el caso de las mujeres. Su efecto es fuerte al tener un coeficiente de -1.0.

Adicionalmente, vemos que los efectos de los resultados del estudio Pisa no son significativos en ninguno de sus tres mediciones (lectura, matemáticas y ciencias) debido a que en cada país los resultados no sufren gran variación de un año a otro.

Para concluir indicar que el R- cuadrado intra grupos es de 0.5335 , lo que nos indica que el 53,35\% de la variabilidad en el teletrabajo es explicada por las variables independientes.

\section{CONCLUSIONES}

1. Del análisis de los resultados del modelo de regresión lineal se obtiene que a un nivel de significación del 5\% un aumento del gasto educativo y una 
mejora del nivel educativo en matemáticas hacen aumentar el porcentaje de teletrabajo en un país. Estos resultados nos permiten pensar que el gasto educativo especialmente centrado en la obtención de mejores resultados en la asignatura de matemáticas impactará positivamente en el porcentaje de teletrabajadores aunque en ambos casos su efecto es muy reducido (ver: Tabla 5: Resultado de la Regresión Lineal).

2. Un dato especialmente interesante que se ha obtenido es que cuanto mayor es el número de trabajadores afiliados en sindicatos, menor es el porcentaje de teletrabajo con un nivel de significación del 5\% (ver: Tabla 5: Resultado de la Regresión Lineal). Este hecho puede ser consecuencia de la idea que se puede tener desde las organizaciones sindicales de poder perder su poder sobre los trabajadores si estos implementan técnicas de teletrabajo.

3. Adicionalmente, se ha realizado un análisis del modelo de efectos fijos en el que los resultados muestran, a un nivel de significación del 1\%, que cuanto mayor es la tasa de desempleo femenino y mayor es el nivel de educación secundaria, menor es el porcentaje de personas que teletrabajan. El resultado de la tasa de desempleo femenino puede ser consecuencia del mayor uso del teletrabajo por parte de las mujeres que de los hombres y por tanto, a mayor tasa de desempleo femenina menor es su porcentaje de teletrabajo (ver Tabla 6: Modelo de Efectos Fijos).

\section{Tabla 6. Modelo de Efectos Fijos}

\begin{tabular}{|c|c|c|c|c|c|c|}
\hline Teletrabajo & Coef. & Std. & Err. & $\mathbf{t}$ & $\mathbf{P}>|\mathbf{t}|$ & {$[\mathbf{9 5 \%}$ Conf. Interval] } \\
\hline Estudio Secundaria & -.5835323 & .1152306 & -5.06 & $0.001 * * *$ & -.8560095 & -.3110552 \\
\hline Estudio Universitario & .0658496 & .1326793 & 0.50 & 0.635 & -.2478871 & .3795862 \\
\hline Gasto Educación & .008668 & .0047137 & 1.84 & 0.109 & -.0024782 & .0198143 \\
\hline Productividad & .8708275 & 17.615 & 0.05 & 0.962 & -40.782 & 42.523 \\
\hline PIB per capita & -16.210 & 18.296 & -0.89 & 0.405 & -59.473 & 27.053 \\
\hline Trabajadores Sindicalizados & .372496 & .2538058 & 1.47 & 0.186 & -.2276594 & .9726513 \\
\hline Desempleo Hombres & .8593475 & .4096769 & 2.10 & $0.074 *$ & -.1093845 & 1.828 \\
\hline Desempleo Mujeres & -1.081 & .1921034 & -5.63 & $0.001 * * *$ & -1.536 & -.6276307 \\
\hline Coste por mega & .0833187 & .0728257 & 1.14 & 0.290 & -.0888866 & .2555241 \\
\hline Resultado Lectura informe Pisa & -.1496475 & .105816 & -1.41 & 0.200 & -.3998627 & .1005677 \\
\hline Resultado Matemáticas informe Pisa & -.0316193 & .03902 & -0.81 & 0.444 & -.1238869 & .0606484 \\
\hline Resultado Ciencias informe Pisa & .0737 & .0725367 & 1.02 & 0.343 & -.0978221 & .245222 \\
\hline Constante & 148.921 & 77.549 & 1.92 & 0.096 & -34.454 & 332.296 \\
\hline
\end{tabular}

Significativo al: *10\%, Significativo al **5\%, Significativo al ***1\%.

Las variables (Productividad, PIB per cápita y Coste por mega) se encuentran definidas en logaritmo. 


\section{BIBLIOGRAFÍA}

AGENTES SOCIALES CES, UNICE/UEAPME Y CEEP (2002), Acuerdo Marco Europeo sobre Teletrabajo. Bruselas, 16 julio 2002.

ALEXANDER, S. y RUDERMAN, M. (1987), "The role of procedural and distributive justice in organizational behavior", Social Justice Research, 1(2), 177-198.

ANDRIESSEN, J. y VARTIAINEN, M. (2006), Mobile Virtual Work. A New Paradigm? Berlin: Springer.

BAILEY, D. y KURLAND, N. (2002), "A review of telework research: findings, new directions, and lessons for the study of modern work", Journal of Organizational Dynamics, núm. 28, 383-400.

BARUCH, K. (2001), "The status of research on teleworking and an agenda for future research", International Journal of Management Reviews, vol 3(2), 113-129.

BENTLEY, K. y YOONG, P. (2000), "Knowledge work and telework: an exploratory study", Internet Research: Electronic Networking Applications and Policy, núm. 10, 346-356.

BLOOM, N. y otros (2013). Does working from home work? Evidence from a Chinese experiment. Stanford University. www.stanford.edu/ nbloom/WFH.pdf

BRODT, T. L. y VERBURG, R. M. (2007), "Managing mobile work-insights from European practice", New Technology, Work and Employment, núm. 22, 52-65.

COUNCIL OF ECONOMIC ADVISORS (2010), "Work-life balance and the economics of workplace flexibility", http://www.whitehouse.gov/files/ documents/100331-ceaeconomics-workplaceflexibility. pdf

COLLINS, M. (2005), "The not so simple case for teleworking: a study at Lloyd's of London", New Technology, Work and Employment, núm. 20, 115-132.

COOPER C. y KURLAND, N. B. (2002), "Telecommuting, professional isolation and employee development in public and private organizations", Journal of Organizational Behavior, 23(4), 511-532.

CYBER DIALOGUE INC. (2000), Small Business/Home Office Trend Report, New York.

DEAKIN, S. y KOUKIADAKI, A. (2007), The capability approach and the reception of European social policy in the UK: The case of the Telework Agreement, Cambridge: Centre for Business Research, University of Cambridge.

DVORAK, J. y ANIS, N (1992), Dvorak's inside track to DOS and PC performance, Osborne: McGraw-Hill.

EUROSTAT (2011), Population in employment working from home as a percentage of the total employment, for a given sex, age group and professional status (\%), http://ec.europa.eu/eurostat. 
EUROPEAN FOUNDATION FOR THE IMPROVEMENT OF LIVING AND WORKING CONDITIONS (2010), Telework in the European Union. information@eurofound.europa.eu.

FENSON, B. y HILL, S. (2003), Implementing and managing telework: A guide for those who make it happen. Westport, CT: Praeger Publishers.

FURNAS, A. (2000), Dealing with co-worker jealousy. Working at home. En http://www.balancedlifedesign.com.

GARCÍA VIÑAS, J. (2001), "Relaciones Laborales e internet”, Revista de Trabajo y Seguridad Social, núm. 223, 3-78.

GOLDEN, T. (2007), "Co-workers who telework and the impact on those in the office: Understanding the implications of virtual work for co-worker satisfaction and turnover intentions", Human Relations, núm. 60 (11), 1641-1667.

GRAY, P (1995). "The virtual workplace," OR/MS Today, vol. 22, núm. 4, pp. $22-26$

GRENIER, J y otros (1997), "Internal versus external labour flexibility: a two plant comparison in Canadian manufacturing”, Industrial Relations, núm. 52, 683-711.

HARRIS, L. (2003), "Home-based teleworking and the employment relationship", Personnel Review, núm. 32, 422-437.

HILl, E. J. y otros (1998), "Influences of the virtual office on aspects of work and work/life balance", Personnel Psychology, núm. 51(3), 667-683.

MARGINSON, P. y SISSON, K. (2006), European Integration and Industrial Relations, Palgrave: Macmillan,

MINISTERIO DE EMPLEO Y SEGURIDAD SOCIAL (2012), Real Decreto-ley 3/2012, de 10 de febrero, de medidas urgentes para la reforma del mercado laboral.

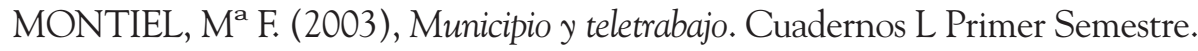

NANDHAKUMAR, J. y BASKERVILLE, R. (2006), "Durability of online networking: patterns of trust", Information Technology 83 People, núm. 19, 371-389.

NILLES, J. M. (1998), Managing Telework. Strategies for Managing the Virtual Workforce, New York, NY: Wiley.

OETTINGER, G. (2012), "The Incidence and Wage Consequences of HomeBased Work in the United States, 1980-2000", Journal of Human Resources forthcoming, 237-260.

PARLAMENTO EUROPEO (2000), Directiva sobre el comercio electrónico 2000/31/CE del Parlamento Europeo, Bruselas: Diario Oficial de la Unión Europea. L 178/1 del 17-7-2000.

PETERS, P. y otros (2001), "Factors in Employees. Telecommuting Opportunities, Preferences and Practices”, Research Paper, núm 008. Paper presented at the 6th International ITF workshop 83 business conference 'Working in the new economy' Amsterdam, August 26th-30 th. 
PYÖRIÄ, P. (2011), "Managing telework: risks, fears and rules", Management Research Review, núm. 34, 386-399.

RACC. (2007). Emisiones de CO2. Marketing i Comunicació Corporativa del RACC, Barcelona.

ROSENLUND, C. (2010), Examining Possible Effects of Telework Upon Career Advancement, San Francisco, CA: San Francisco State University.

WATAD, M. y DISANZO, F. J. (2000), "Case study: the synergism of telecommuting and office automation", Sloan Management Review, núm. 41, 85-96.

WELZ, C. y WOLF, F. (2010), Telework in the European Union. Eurofound. Disponible en: http://eurofound.europa.eu/observatories/eurwork/comparative-information/telework-in-the-european-union 